Accountability in Research, 2000. 8: p. 55. Reprinted with permission of publisher Taylor and Francis.

\title{
CHASING ANOMALOUS SIGNALS: THE COLD FUSION QUESTION
}

\author{
S.E. Jones \\ Department of Physics and Astronomy \\ Brigham Young University \\ Provo, Utah 84602
}

What should a scientist do with an anomalous experimental result (one which flies in the face of prevailing theories), which is statistically significant (at the five sigma + level), yet which cannot be repeated at will? This is the problem which plagued us early on in our non-plasma or "cold" fusion experiments which began in spring 1986 at Brigham Young University, 2.5 years before we heard of the ostensibly-related work of Drs. Pons and Fleischmann. It is a question which haunts us still. I invite the reader to seriously consider the question posed above: what would you do with such data? If you walk away from an anomalous result, you could miss something important. Indeed, is it scientifically honest to ignore such data? One can argue that scientific instruments often show "glitches," and this is probably just one of these. Quite possibly. But what if the "glitch" is not only statistically significant, but also has interesting characteristics, such as the correct energy $(2.5 \mathrm{MeV})$ for neutrons from deuteron-deuteron fusion, and the expected signal-width? [1] What if other data runs which are individually significant only at the 2-sigma level also contribute this peak? [1]

Well, what would you do? Keep trying to find the trigger mechanism, if any? How many months would you be willing to spend/waste? Would you continue if all grants dried up for this project and if virtually everyone thought the effect was non-existent and laughable?

In December 1991, a group of scientists assembled at Half Moon Bay in California (organized by Nathan Hoffman and the Electric Power Research Institute) to discuss our research on low-level nuclear products from deuterided materials. The group listened while I described recent results from BYU and from our experiments at the Kamiokande in Japan. Howard Menlove discussed results from Los Alamos National Laboratory. Ed Cecil showed chargedparticle signals from the Colorado School of Mines. Kevin Wolf from Texas A\&M showed puzzling nuclear data, too. V. Tsarev reviewed data from Russia. Yes, we had tantalizing results, but these were tiny signals and not reproducible at will.

I fully expected the scientists from Cal Tech and the University of Pennsylvania and elsewhere to simply recommend that the work stop. A few of them later told me that is what they had anticipated doing. But they did not. Instead, they recommended that we look again, with specific pointers of how to improve our detectors and eliminate possible spurious effects. So we proceeded. We set up a new laboratory deep in an underground tunnel in the mouth of Provo Canyon, about $10 \mathrm{~km}$ from the BYU campus. Under many tons of rock, we greatly reduced the background due to cosmic rays. We also placed three large scintillation counters around our system to actively veto cosmic rays. 
The primary detector involved sixteen helium-3-filled proportional counter tubes in four groups of 4 counters each. The idea behind this segmentation was that the distribution of neutrons ought to be isotropic from real events and this would be testable given sufficient statistics. On the other hand, a spurious "burst" of neutrons would likely show up in just one segment of the detector.

Inside this detector we placed a plastic-scintillator core viewed by a photomultiplier tube. Fast neutrons from the sample often generate a recoil proton in the plastic, generating scintillations viewed by the fast PMT. We required a delayed coincidence between the prompt scintillator pulse and the neutron-capture signal from a $3 \mathrm{He}$-tube. Background is thereby reduced to about 0.7 counts per hour while the $2.5-\mathrm{MeV}$-detection efficiency is about $9 \%$. The good neutron detection efficiency coupled with low background is comparable to the best in the world, that of the Kamiokande detector (which is now dedicated to neutrino detection).

Following the advice of Al Mann and Charles Barnes, we also digitized all signals using a LeCroy fast-waveform digitizer, so that we retain pulse-shape information as well as timing between pulses. Pulse-shape analysis permits excellent noise rejection, along with giving neutron-energy information from the prompt plastic scintillator pulses. By rejecting events having small or no scintillator pulses, we strongly discriminate against slow neutrons. This background-reducing feature is not available to most detectors, including the Kamiokande and BF3 detectors.

We were ready. Right away, we found large "bursts" that were in fact spurious. The characteristic of these "bursts" is that the signals all appear in the same quadrant of the 3-Helium detector, while nothing is seen at all in the plastic scintillator. With the help of Howard Menlove, we determined that these large multiplicity "events" are caused by high-voltage breakdown in the standard electronics of this 3He-type detector. The "events" are spurious. The Los Alamos detectors of this type are susceptible to this problem, and generally do not have the segmentation necessary to reveal the spurious nature of the signals. Our previous claims to "neutron burst from cold fusion" were retracted. [2] Ouch.

We were glad to have learned a valuable lesson: if you are going to chase an anomalous signal, you had better use the best detectors possible!

It never ceases to amaze me how many workers in the "field of cold fusion" persist in using crude instruments which are likely to give misleading "signals". Some researchers continue to use x-ray films instead of x-ray spectrometers, helium or tritium gas sampling instead of charged-particle spectrometers, geiger counters rather than silicon or germanium detectors, and neutron survey meters instead of sensitive neutron detectors such as described above. It is disquieting that some researchers select open electrolytic cells over closed cells, and excessively long sampling intervals (e.g., 5-minute sampling intervals for input voltage used by Pons and Fleischmann in calculating excess heat over a 10-minute boiling period [3]).

It is high time to strongly question claims of cold fusion based on crude techniques and to demand tests at a rigorous scientific- proof level. Compelling evidence requires use of the best instruments available, incorporating fast data-sampling and digitization methods, for instance. Different detectors must give signals which agree quantitatively. A real signal will be capable of scaling, and should not shrink as background levels are reduced. Otherwise, the researcher may well be chasing noise, and probably making noise as well. (Hyping questionable results in the 
media seems to be a characteristic practice of those claiming excess heat, which we never did.) I have not seen any compelling evidence for any "cold fusion" effects, to date.

Meanwhile, it is true that we at BYU are quietly continuing to pursue the possibility of low-level fusion in deuterided materials, although I do not usually advertise this fact. It is all somewhat embarrassing and very frustrating. We are looking for fusion associated with sonoluminescence in collapsing deuterium bubbles, among other approaches.

We continue to see possible neutron signals from time to time, at the three-to-five standard-deviation level, in our best segmented detector, deep underground. These results do not appear to be due to electrical noise or detector glitches or cosmic rays or contamination, or anything else we can think of (so far). We cannot now, however, produce these signals at will. Until we can, we will not say much. We have invited -- challenged -- other researchers to bring their "cold fusion" devices here for testing. Few have accepted our offer and none have succeeded in generating a reproducible signal. Yet as long as students here have any interest, we will probably keep beating our heads against this -- but using state-of-the-art instruments!

\section{REFERENCES}

1. S.E. Jones, E.P. Palmer, J.B. Czirr, D.L. Decker, G.L. Jensen, J.M. Thorne, S.F. Taylor and J. Rafelski, "Observation of Cold Nuclear Fusion in Condensed Matter," Nature, 338 (1989) 737.

2. S.E. Jones, D.E. Jones, D.S. Shelton, and S.F. Taylor, "Search for Neutron, Gamma and Xray Emissions from Pd/LiOD Electrolytic Cells: A Null Result," Trans. Fusion Tech. 26 (1994) 143.

3. M. Fleischmann and B.S. Pons, "Calorimetry of the Pd-D2O system: from simplicity via complications to simplicity," Phys. Lett. A 176 (1993) 176; and "Fleischmann respond to Jones, " 28 October 1993 posting on sci.physics.fusion. 